\title{
Design and Geodetic Adjustment of Levelling Network for Establishment of Unified Control Points in Korea
}

\author{
Lee, H. K., ${ }^{1}$ Yun, S. H., ${ }^{2}$ Kwon, J. H. ${ }^{3}$ and Lee, J. S. ${ }^{4}$ \\ ${ }^{1}$ The school of Civil, Environmental and Chemical Engineering, Changwon National University, 20 \\ Changwondaehak-ro, Uichang-gu, Changwon, Gysangnam-do, 51140, Republic of Korea \\ E-mail: hkyulee@changwon.ac.kr \\ ${ }^{2}$ The Department of Echo-Friendly Offshore FEED Engineering, Changwon National University, 20 \\ Changwondaehak-ro, Uichang-gu, Changwon, Gysangnam-do, 51140, Republic of Korea \\ E-mail: shyun92@changwon.ac.kr \\ ${ }^{3}$ The Department of Geoinformatics, The University of Seoul, Republic of Korea, 163 Seoulsiripdae-ro, \\ Dongdaemu-gu, Seoul, 02504, Republic of Korea, E-mail: jkwon@uos.ac.kr \\ ${ }^{4}$ The Department of Geoinformatics, The University of Seoul, 163 Seoulsiripdae-ro, Dongdaemu-gu, Seoul, \\ 02504, Republic of Korea, E-mail: leejs@uos.ac.kr
}

\begin{abstract}
The unified control points (UCPs), the multi-dimensional geodetic reference points installed in the low elevated area, has been established in Korea for the combination of the horizontal and the vertical geodetic control. While the $1^{\text {st }}$-phase UCPs network was completed in 2011 with a spatial density of about 10km, its densification has been underway as the $2^{\text {nd }}$-phase project. The UCPs supersedes the legacy geodetic points, such as triangulation points and benchmarks (BMs) once the $2^{\text {nd }}$-phase implementation. Although the horizontal network of the UPCs can be readily realised by GNSS technology, a technical challenge of the vertical component remains to be settled due to characteristics of the geodetic levelling technique. To this end, a pilot study was conducted to design a new version of the UCPs-based levelling network and to demonstrate its effectiveness with a comparison of the legacy. In this paper, a concept of the UCPs-based levelling network is briefly addressed with some prominent examples, and details of a pilot network and its measurements is given with the network adjustment procedure. Finally, results of the adjustments are provided with an emphasis on the influence of the newly designed network in terms of accuracy, reliability and estimated heights.
\end{abstract}

\section{Introduction}

Since the early $20^{\text {th }}$ century, triangulation points (TPs) and levelling benchmarks (BMs) have played an indispensable role in the geodetic reference in Korea. After the adoption of the Korean geocentric datum 2002 (KGD2002) in 2003, which was aligned to the international terrestrial reference frame 2000 (ITRF2000), the nationwide geodetic adjustments of the TPs and BMs were completed in 2006 for the densification (NGII, 2006 and Lee et al., 2008). Note that the TPs had been mostly re-surveyed by the modern GNSS technique whereas the latest geodetic levelling observations were included to the adjustment. These geodetic efforts led to the successful modernisation of the Korean geodetic infrastructure in terms of accuracy and compatibility with the international standards. Nevertheless, the location of the TPs and the spatial density of the $\mathrm{BMs}$ restricted their practical usage; hence, the national geographical information institute of Korea (NGII), the geodetic and mapping agency, has designed and established the so-called the unified control points (UCPs) as a multi-dimensional geodetic framework by publishing he geodetic latitudes and longitudes, ellipsoidal heights, geoidal undulations, and gravity anomaly (Bae et al., 2011). Being installed in the low elevated areas, the UCPs generally have a high potential to significantly improve the accessibility and usability (NGII, 2013).

As the $1^{\text {st }}$-phase, a total of 1,196 UCPs (hereafter, the $1^{\text {st }}$-phase UCPs) had been installed at around $10 \mathrm{~km}$ gridding spaces and surveyed by GNSS receivers, spirit-levels and gravimeters for three years since 2008. The GNSS measurements were simultaneously adjusted to estimate the 3-D geodetic coordinates. On the other hand, the normal orthometric heights of the UCPs were determined by a simple arithmetic computation as they were only connected to one side of the nearest BMs by the double-running surveys. Even though the $10 \mathrm{~km}$ gridding was dense enough to support the modern GNSS surveys, it was still far sparse for other 
aditional surveying techniques, such as the traversing and especially the spirit-levelling. To mitigate this situation, NGII began with the $2^{\text {nd }}$ phase implementation of UCPs (hereafter, the $2^{\text {nd }}$ phase UCPs) in 2012 for densification of the $1^{\text {st }}$ phase control up to $3 \mathrm{~km}$ gridding intervals. It is crucial to be noted that the nationwide geodetic levelling network has also been rebuilt as part of the project by an application of a new design that combines the UCPs and the BMs loops. As a consequence, the new geodetic framework (i.e., the densified UCPs network) is expected to supersede the legacy geodetic networks after the full implementation (NGII, 2015).

For the establishment of the UCPs infrastructure, geodetic adjustments of the GNSS and geodetic levelling observations should be conducted to determine the reference coordinates to be published. With the characteristic of the modern GNSS surveying technology, the adjustment can be readily completed. On the other hand, the vertical network is facing some technical challenges, including a completely new design of the levelling loops and resurveys of the $1^{\text {st }}$-phase UCPs. In order to appropriately cope with this issue, a pilot study has been performed to design a UCPs-based levelling network with which integrated the existing BMs and to gain some practical experience of the geodetic adjustments that can be a basis of the strategy and procedure development. This paper firstly introduces a design concept of the network with three approaches and subsequently provides the pilot network designed in this study. After then, some results of the geodetic network adjustments are given with emphasis on the impact of the newly designed network in terms of accuracy, reliability and estimated heights.

\section{Design of a Pilot Leveling Network for UCPs}

\subsection{Concept and Strategy}

The UCPs project has been underway for the maximum density of about $3 \mathrm{~km}$ except for the mountainous area. Note that the goal is ultimately not only to replace the legacy geodetic points (e.g., TPs and the BMs) but to construct a single-layer geodetic network; hence, usability, accuracy and reliability of the national geodetic infrastructure are enhanced. To achieve this goal, both of the GNSS and the geodetic levelling network should be rebuilt and adjusted, so as to accurately and reliably estimate reference coordinates. However, this research has only focused on the latter since the former can be efficiently conducted by modern GNSS technology.

Some technical issues related to the geodetic levelling and the geometry of the $1^{\text {st }}$-phase UCP's levelling loops should be considered for design of the levelling network. For instance, the surveying method itself generally requires substantial laborious works, and a loop of the $1^{\text {st }}$-phase UCP is only linked to the nearest single BM; therefore, it is advisable to include some of the BM's loops in the new network to reduce the re-surveying burden and to strengthen geometry of the $1^{\text {st }}$-phase UCP's levelling loops. To deal with these problems, three strategies were derived and employed in this study: (a) replacement of BMs by UCPs; (b) designation of BMs as UCPs by GNSS surveys; (c) new installation of the $2^{\text {nd }}$-phase UCPs.

As shown in Figure 1, the $1^{\text {st }}$-phase UCPs are only connected to a single BM, e.g., see, dotted lines, whereas the $2^{\text {nd }}$-phase UPC is linked to at least two BMs, denoted by the solid lines. Considering $3 \mathrm{~km}$ radius of the solid grey circles, UCPs can replace some of $\mathrm{BMs}$ with keeping up with the target density. For instance, the BM '01-00-00' and '01-01-01' can be excluded if the loop '01-01$02 \leftrightarrow \mathrm{UCP} 1 \leftrightarrow$ 01-02-01' is created by a field campaign; their connecting loops have no reason to be maintained in the new configuration. By adding a 'UCP2' and connecting it to the two nearest points, the levelling loop between the BMs (e.g., 01-02$02 \leftrightarrow 01-02-03)$ can be excluded from the network. Furthermore, surveying a line between 'UCP2' and '01-04-00' indeed enhance the geometric strength of the network.

Figure 2 depicts the concept which designates the existing BMs for the UCPs by GNSS surveys. No UCP is found in the grey-coloured grids in the figure, but at least one UCP should be installed within each of them to fulfil the target density of around $3 \mathrm{~km}$. In this case, field reconnaissance process is carried out to look for candidates of the designated UCPs, namely the BMs having benign GNSS operational environment. As denoted in Figure 2, if possible, the BM '01-00-00' and '01-0203 ' can be switched to the UCPs by surveying GNSS and publishing the 3-D geodetic coordinates (i.e., latitude, longitude, and ellipsoidal height). To this end, no UPC is installed in the $2^{\text {nd }}$ row and $1^{\text {st }}$ and $3^{\text {rd }}$ column of the grid system in Figure 2; however, at least one UCP should be added in the $2^{\text {nd }}$ row and $2^{\text {nd }}$ column. On the other hand, Figure 3 shows an example of the case that a new UCP is installed in the UCPs-empty grid, denoted by greycoloured. After the field reconnaissance, a final decision of the location is made with a consideration of surveying efficiency. Note that the levelling loops of the new UCPs must be connected to more than two control points. 


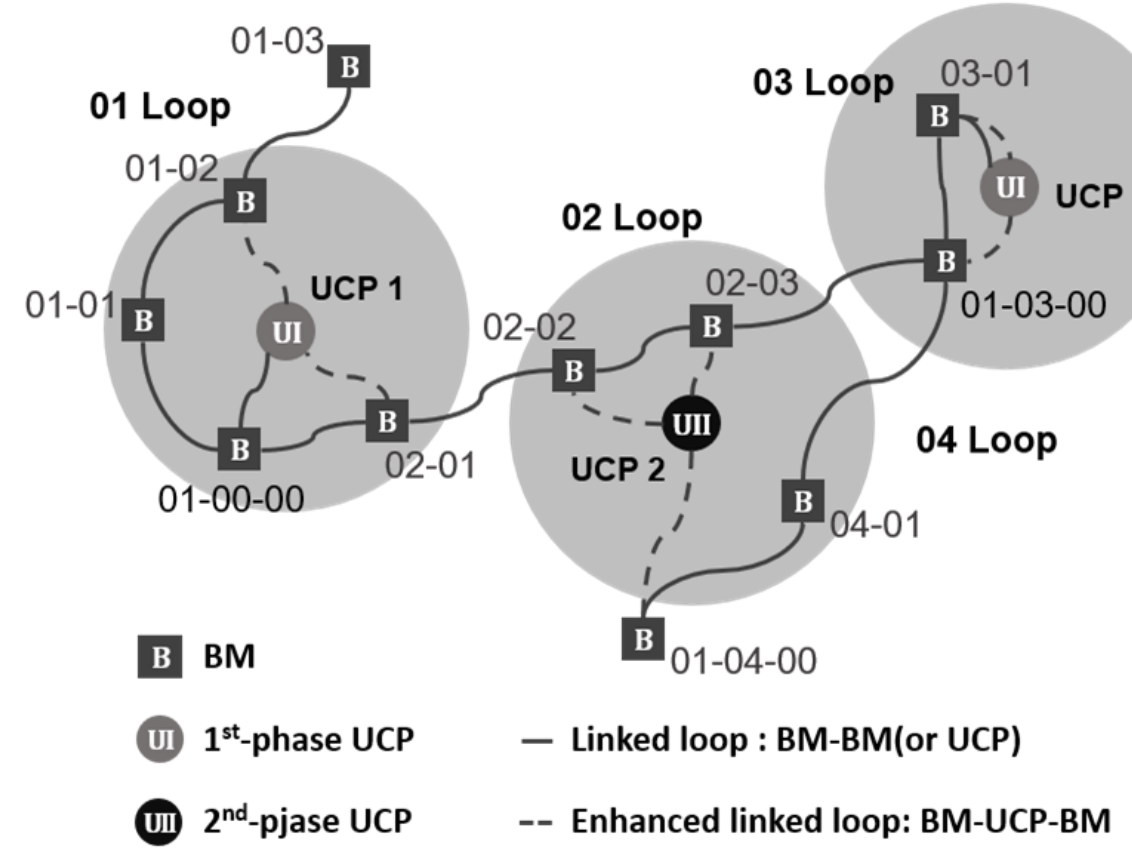

Figure 1: Replacement of BMs by UCPs for the design of the UCPs-based levelling network

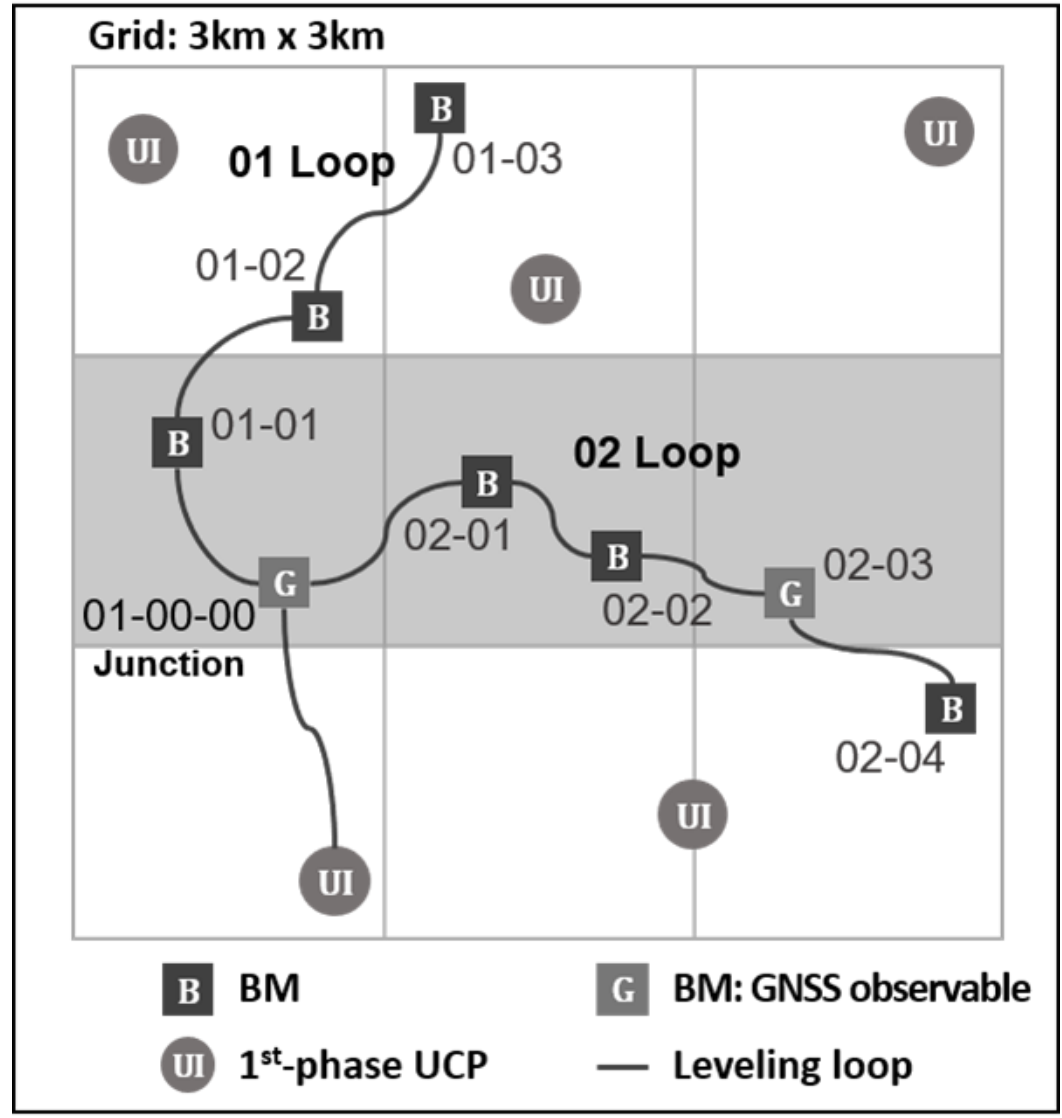

Figure 2: Designation of BMs as UCPs for the levelling network design 


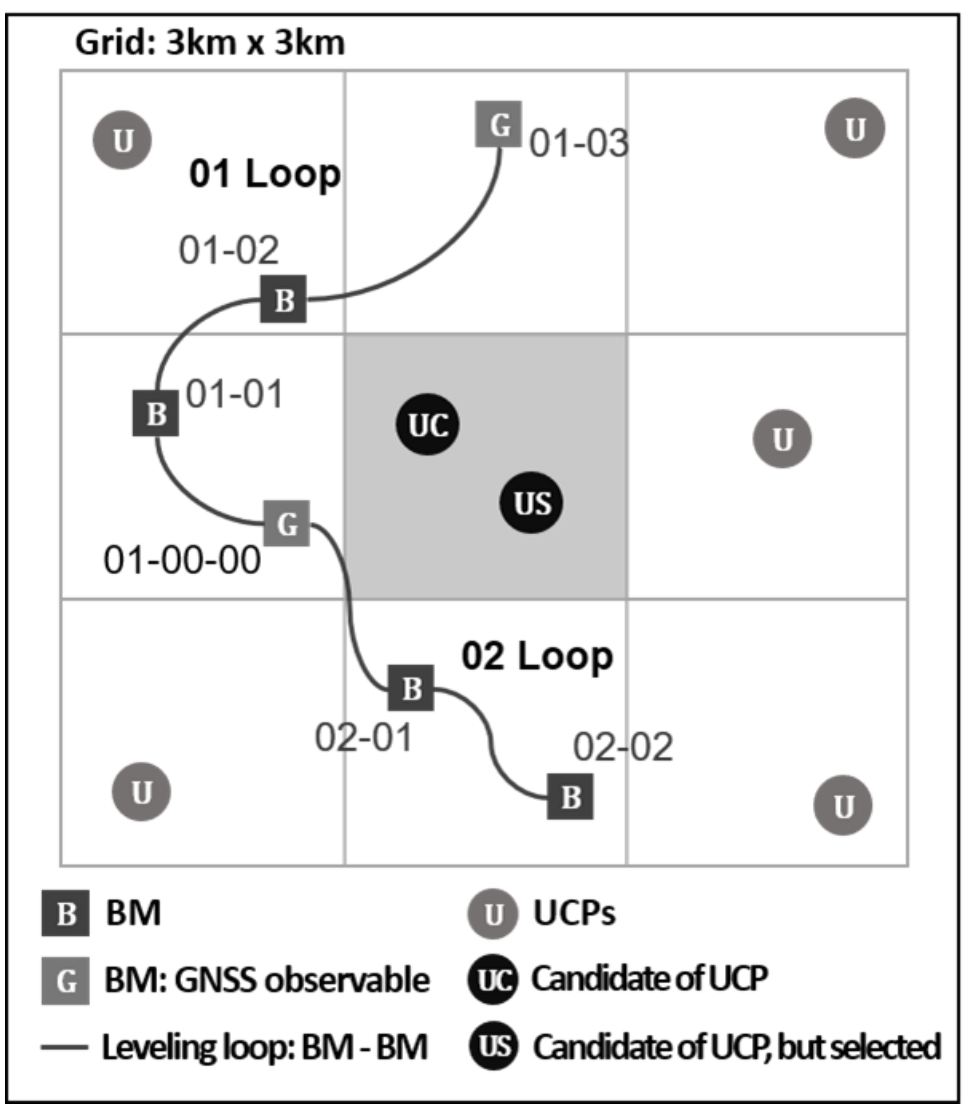

Figure 3: Installation of new UCPs for the levelling network design

\subsection{Design of a Pilot UPCs-based Levelling}

Network

The $12^{\text {th }}$-circuit of the $1^{\text {st }}$-order geodetic levelling loops was selected in this study to design and establish a pilot network, including the national vertical origin. Figure 4 shows the pilot levelling network designed by the application of the concepts mentioned above. Note that the eight the UCPs along the west shoreline were newly installed by this project to enhance the geometric strength of the network, and the five levelling sections in an inland area were surveyed, e.g., see Figure 4. As given in Table 1 , the network is consisted of 621 points, but only $34 \%$ of them is the newly installed UCPs. It is, however, worth understanding that NGII has a short-term plan: (a) to install more the UCPs for the spatial density enhancement; (b) to re-survey the $2^{\text {nd }}$-class BM loops by the $1^{\text {st }}$-class standard; (c) to conduct GNSS campaign for the designation of selected BMs for UCPs.

Figure 5 is a magnified view of a portion of Figure 4 to present features of the pilot network (i.e., the right picture) with a comparison of the legacy version (i.e., the left picture), which is only comprised of the $1^{\text {st }}$-phase UCPs and the BMs.
Examining the left network in the figure, all the $1^{\text {st }}$ phase UCPs are only connected to a single BM as previously mentioned, e.g., see U290. On the other hand, the right network in the same figure shows that the number of control points is increased by adding the $2^{\text {nd }}$-phase UCPs, and all the UCPs are linked to at least the two near points. It is, also, of interest to observe the UCP290 inside the dotted circle in Figure 5 in that it has been replaced the nearest BM and serves as a junction of the levelling network. Besides, the UPCs inside dotted rectangular on the right picture in the figure are the BMs designated as the UCPs.

\section{Geodetic Adjustment of UCPs-based Levelling Network}

\subsection{Procedure}

A procedure of geodetic adjustment for the pilot network adjustment is shown in Figure 6, which mainly consists of the three steps: (a) examination of the loop misclosure; (b) preliminary adjustments for troubleshooting of outliers and refinement of a stochastic model; (c) final adjustment to estimate normal orthometric heights. 


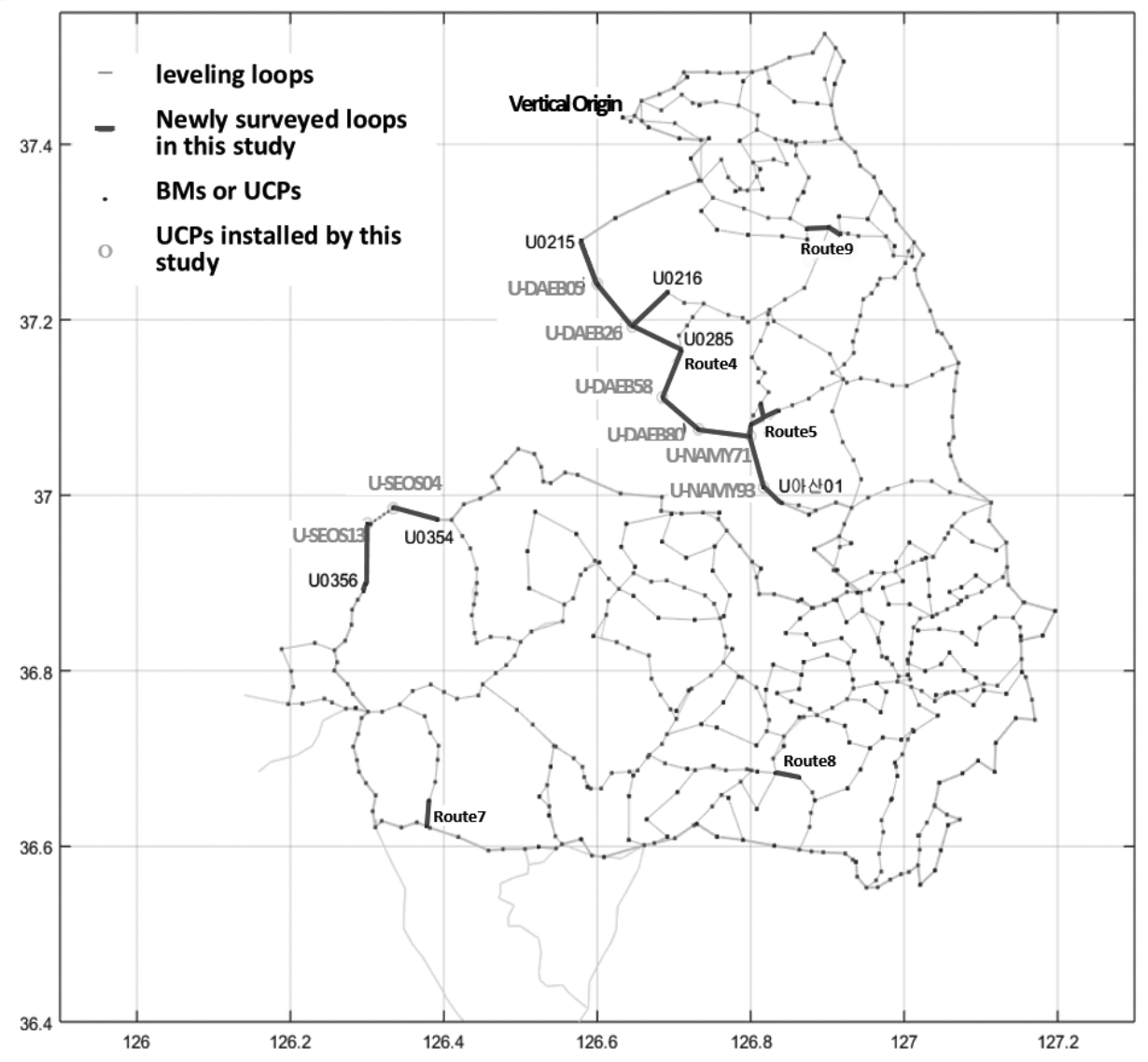

Figure 4: A pilot UCPs-based levelling network

Table 1: The number of geodetic control points included in the pilot network

\begin{tabular}{|c|c|c|c|c|c|}
\hline \multicolumn{2}{|c|}{ BMs } & \multicolumn{2}{|c|}{ UCPs } & \multirow{2}{*}{$\begin{array}{l}\text { UCPs installed by } \\
\text { this project }\end{array}$} & \multirow{2}{*}{ Total } \\
\hline $1^{\text {st }}$-order & $2^{\text {nd }}$-order & $1^{\text {st }}$-phase & $2^{\text {nd }}$-phase & & \\
\hline 105 & 307 & 17 & 182 & 10 & 621 \\
\hline
\end{tabular}

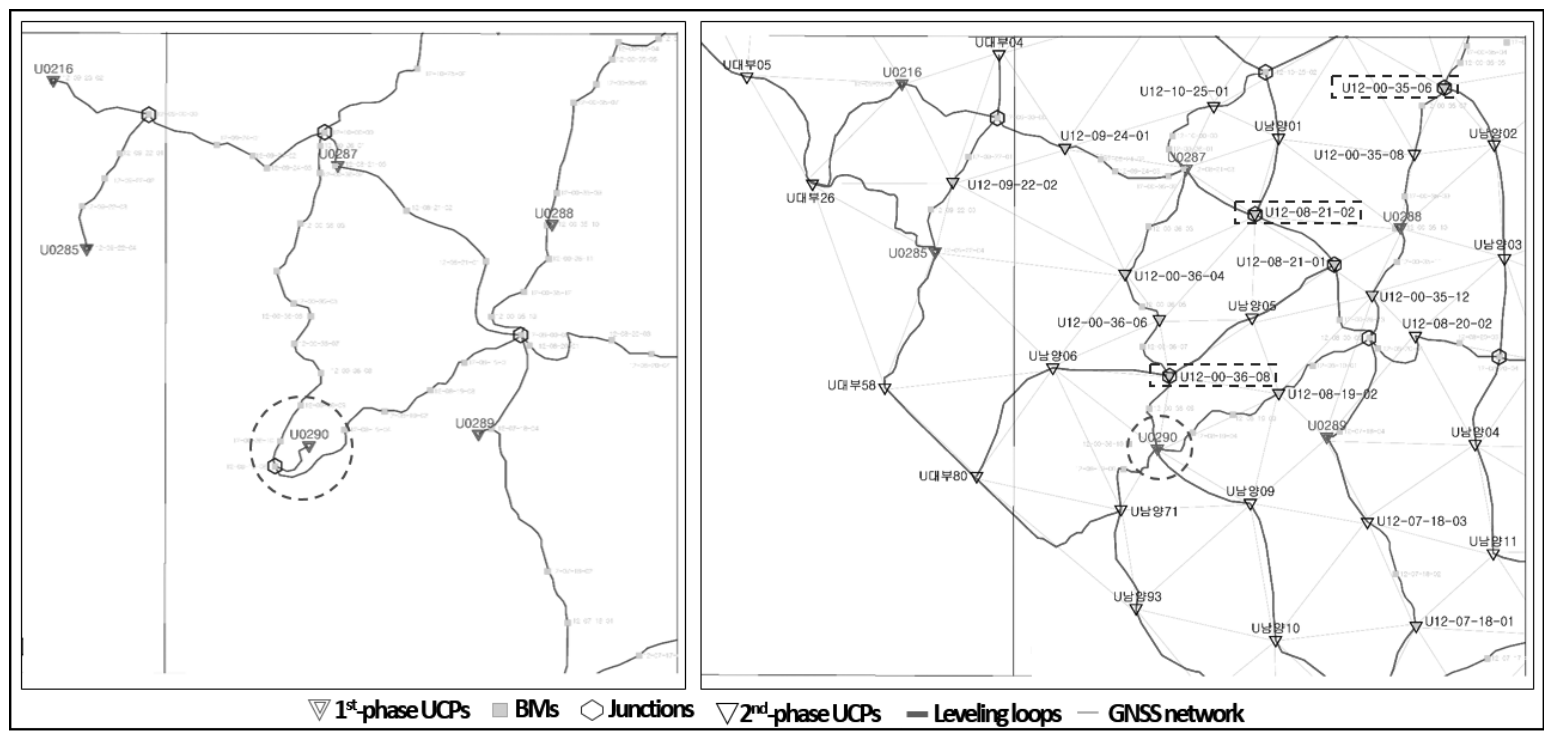

Figure 5: A comparison of the pilot levelling network with that of the legacy 


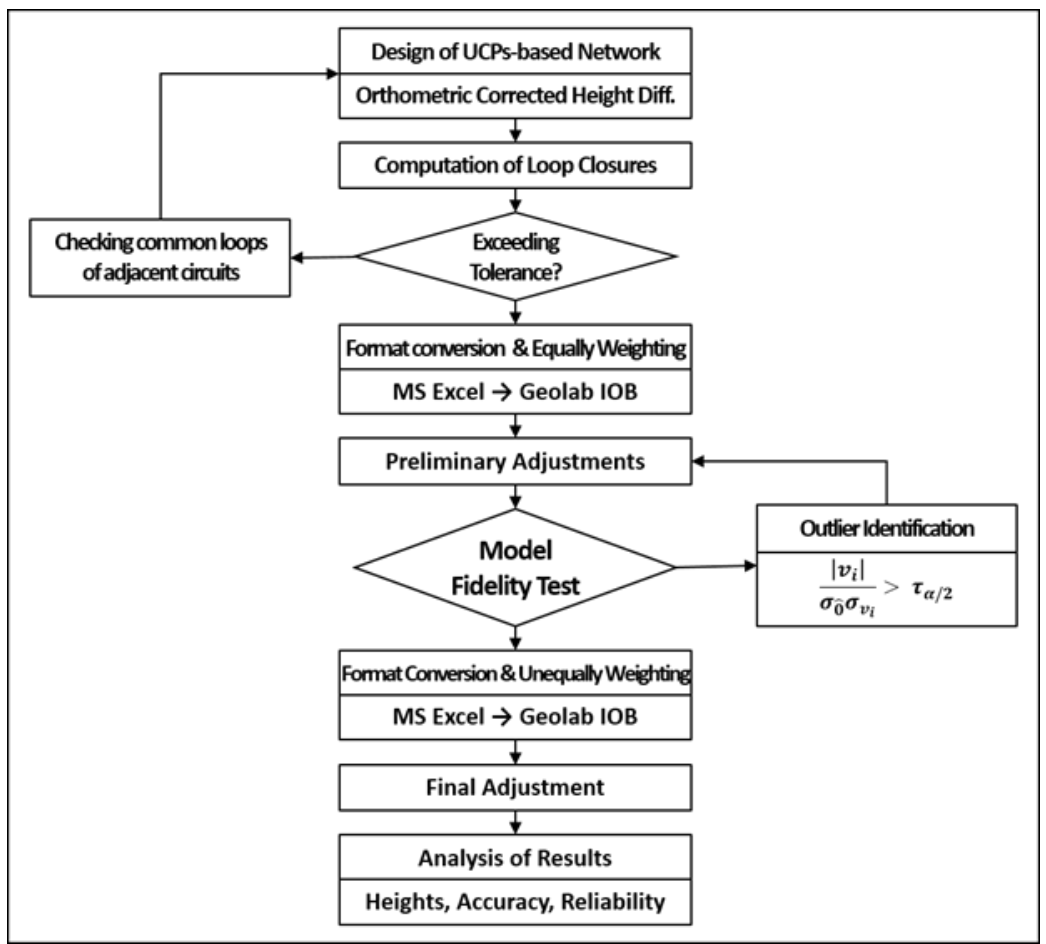

Figure 6: A procedure of geodetic adjustment for the pilot network

Table 2: Statistical summary of the loop misclosures

\begin{tabular}{|l|c|c|c|c|}
\hline Items & Max. & Min. & Mean & Standard Dev. \\
\hline Misclosures $(\mathrm{mm})$ & 76.3 & 0.1 & 15.3 & 16.4 \\
\hline $\begin{array}{l}\text { Normalised misclosures } \\
(\mathrm{mm} / \sqrt{\mathrm{Km}})\end{array}$ & 22.1 & 0.0 & 2.3 & 3.1 \\
\hline
\end{tabular}

Note that the normal gravity was corrected to geometric height differences obtained by geodetic levelling (Bomford, 1962) because gravity surveying over the network had not been completed at the time of this study. However, the future UCPsbased network will adopt the so-called orthometric height system after completing the gravimetric surveys.

It is well known that pre-analysis of measurements is exceptionally critical for geodetic levelling loops to deal with possible outliers as the degree of freedom (DoF) is limited in general. As the first step, loop misclosures were computed and compared to the NGII specifications. Since GeolabPX5 software by BitWise Idea Inc. was used in this study, MS-excel formatted observations were converted to Geolab's text file (i.e., IOB) (Steeves, 2015). Subsequently, a series of preliminary adjustments were carried out by fixing the vertical origin to further identify blunders based on $\tau$-test as well as to determine a reference variance for refining a stochastic model (Cross 1994 and Ghilani, 2010). After applying the reference variance to measurements depending on survey classes, final adjustments were conducted twice by using different observation sets: (a) the one described in section 2.2, denoted by CASE-I; (b) the other that the measurements obtained by this study were excluded from the CASE-I (i.e., CASE-II). Finally, analysis of the adjustment outcomes was made to assess accuracy, reliability, and estimated height via a comparison of the two cases.

\subsection{Examination of Loop Misclosures}

A total of 70 levelling circuits was composed, e.g., see Figure 7 and the loop misclosures were computed. As summarised in Table 2, the mean and the standard deviation of the closures is $15.3 \mathrm{~mm}$ and $\pm 16.4 \mathrm{~mm}$, respectively. Such large values were attributed to the loop lengths ranging from $7.7 \mathrm{~km}$ to $560 \mathrm{~km}$; the longer surveying distance, the larger misclosure expected in geodetic levelling. On the other hand, statistics of the normalised misclosures scaled by the distances become reduced, as given Table 2. As the upper graph in Figure 8 indicated some outliers in the standardised misclosures, for 
xample, circuit No. 11, a comparison of them with the tolerances of the $2^{\text {nd }}$-class standard (i.e., $5.0 \mathrm{~mm} /$ $\sqrt{K m}$ ) was made, discovering six circuits exceeded in the maximum allowance. After the careful examination together with the adjacent circuits sharing the suspected loops, some of the inner circuits were revised by eliminating erroneous observations; No. 11 and 29 were merged with No. 7 and 25 , respectively. As a result, the number of circuits exceeding $2^{\text {nd }}$-class tolerance was reduced by half; the averaged misclosure and the normalised misclosures become small.

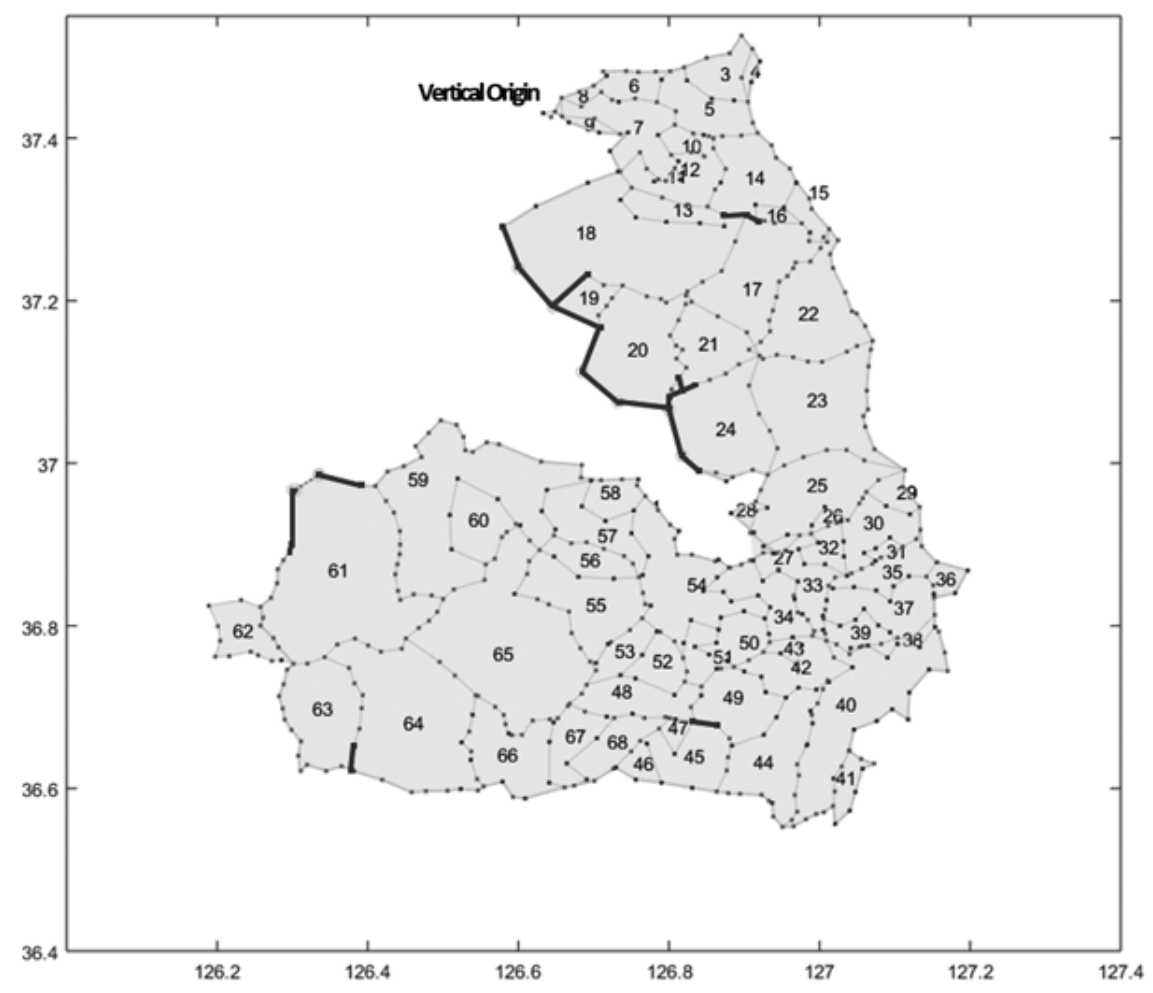

Figure 7: Layout of levelling circuits for checking misclosures

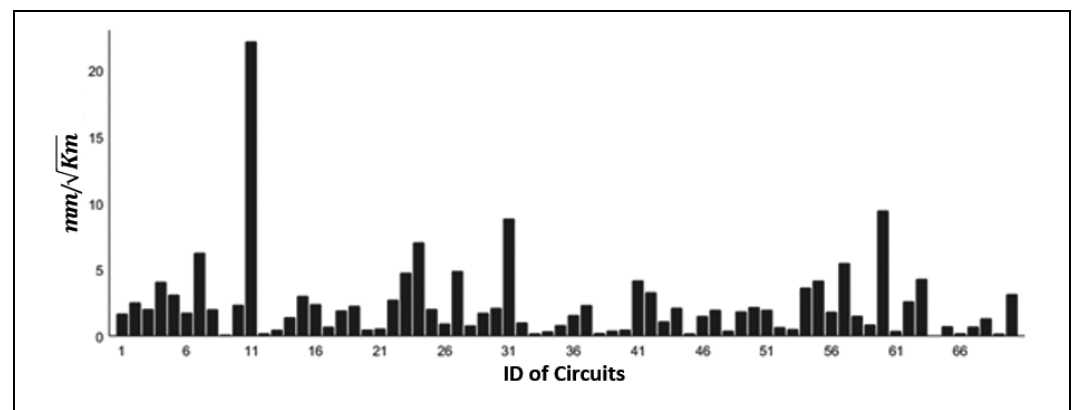

(a) Initial circuits

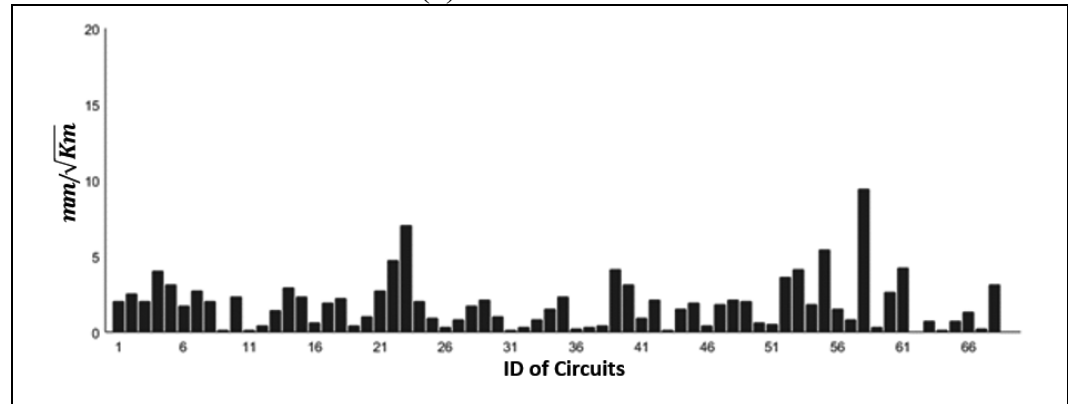

Figure 8: Standardised misclosures of the loop misclosures 
Preliminary Adjustments

A series of network adjustments by holding fixed the national vertical origin were carried out to troubleshoot blunders and to determine a reference variance for stochastic modelling. By referring to NGII (2014), a provisional standard deviation $\pm 2 \mathrm{~mm} / \sqrt{K m}$ was initially assigned to this process. As given in Table 3, the $1^{\text {st }}$-round adjustment resulted in a rejection of the null hypothesis of the model fidelity via a $\chi^{2}$-test (i.e., global test) against a posterior variance. Subsequently, a $\tau$-test (i.e., local test) against the largest residual revealed that the loop 'U-ASAN06 $\leftrightarrow$ BM-12-00-27-18' was a blunder. After excluding it, the $2^{\text {nd }}$-round was subsequently adjusted, yielding a failure to reject the null hypothesis of the global test.

As previously mentioned, the ultimate objective of the UCPs-based network is to establish a signal layer geodetic network with the uniform accuracy of the $1^{\text {st }}$ class survey; however, this pilot network contains 247 measurements of the $2^{\text {nd }}$ class (i.e., $5.0 \mathrm{~mm} / \sqrt{\mathrm{Km}}$ ) as re-survey campaign is still ongoing. In order to reflect this situation in the stochastic model, an empirical approach was employed in this adjustment to derive a reference variance against each a surveying class. That was, an iterative adjustment by varying an initial variance until a hypothesis test against a posterior variance was passed. Note that half of the $2^{\text {nd }}$ class variance was initially assigned to the $1^{\text {st }}$ class according to the NGII standard. Several trials determined $\pm 1.7 \mathrm{~mm} / \sqrt{K m}$ and $\pm 3.4 \mathrm{~mm} / \sqrt{K m}$ as a reference of the $1^{\text {st }}$ and the $2^{\text {nd }}$ class measurements, respectively. Application of these values to an adjustment resulted in a posterior variance 0.981, passing the global test. Figure 9 depicts the relative accuracy of the adjusted measurements: the boxes are results of the equal weighting by $\pm 2.2 \mathrm{~mm} /$ $\sqrt{K m}$; the diamonds indicate those of the unequal weighting by the iterative scheme. Note that the averaged accuracy of the adjustments is somewhat similar (e.g., $3.4 \mathrm{~mm}$ and $3.3 \mathrm{~mm}$ ), but the distinctive standard deviations are seen, namely $\pm 1.4 \mathrm{~mm}$ for the equal weighting and $\pm 0.9 \mathrm{~mm}$ for the unequal weighting. These results are reasonable as the stochastic model with the unequal weighting scheme can more realistically represent the accuracy by reflecting the actual quality of the measurements.

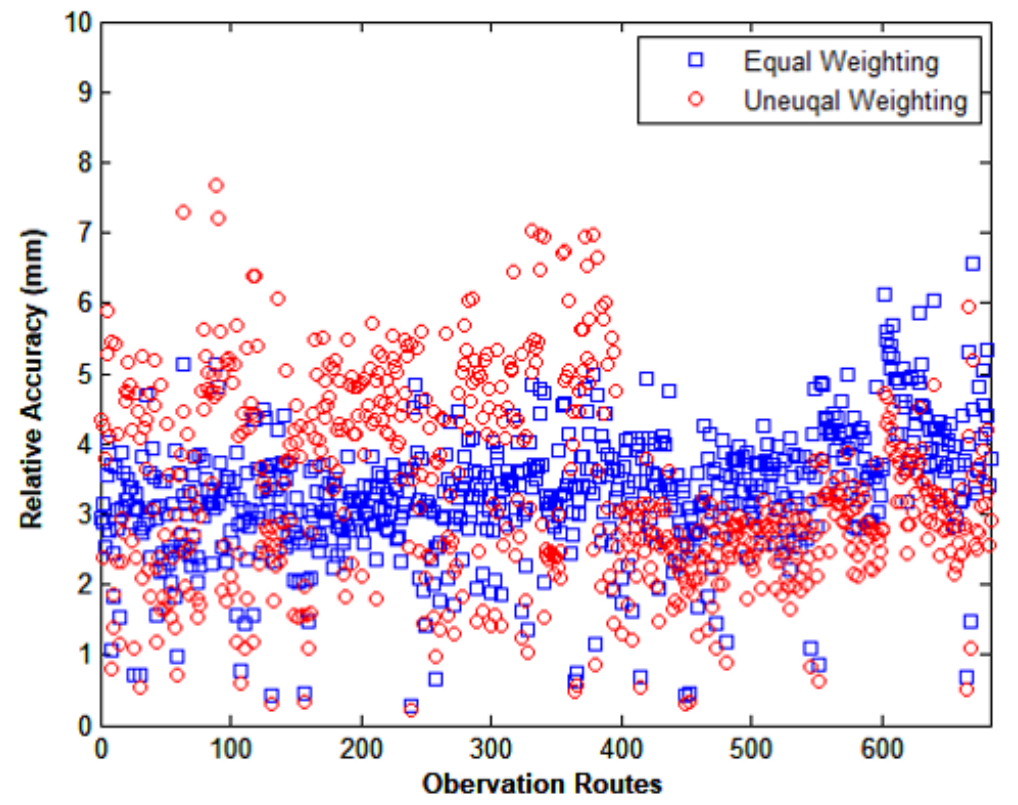

Figure 9: Relative accuracy of the levelling routes

Table 3: Summary of the network adjustment to identify outliers

\begin{tabular}{|c|c|c|c|c|c|}
\hline Trial & \multirow{2}{*}{ No. of points } & No. of Obs. & No. of unknown & $\begin{array}{c}\text { Degree of } \\
\text { freedom }\end{array}$ & $\begin{array}{c}\text { A posterior } \\
\text { variance }\end{array}$ \\
\hline \multirow{2}{*}{$1^{\text {st }}$} & \multirow{2}{*}{618} & 684 & \multirow{2}{*}{617} & 67 & 1.627 (fail) \\
\cline { 1 - 3 } $2^{\text {nd }}$ & & 683 & & 66 & 1.248 (pass) \\
\end{tabular}




\section{Final Adjustments}

As a final process, adjustments were conducted twice by slightly different measurement sets: (a) the observations described in section 3.3, denoted by CASE-I; (b) those same as CASE-I except for the fact that the measurements surveyed by this study were excluded (i.e., CASE-II). The main reason for this approach was to investigate the impact of the new measurements on the accuracy and reliability of the final solution.

\subsubsection{Absolute accuracy}

As shown in Table 4, the final adjustments all passed the model fidelity tests about a posterior variance. It is of interest to see that CASE-I's DoF is more significant than that of CASE-II even though the number of unknowns is reduced.

This is attributed from the fact that some open loops of the traditional BMs-based network along the west coastline were surveyed and connected in the new design, e.g., see Figure 5. While Figure 10 shows the absolute accuracy of the estimated heights, Table 5 summarises the statistics. It can be observed from results that the absolute accuracy has been overall improved by the connection. For instance, the accuracy of the circled area on the right map in Figure 10 is relatively weak as the loops are mostly open; residual errors cannot be distributed by the adjustment over the network. In addition, results of the CASE-I show a clear trend that the accuracy is deteriorated as the control points are becoming away from the datum origin, which is actually a drawback of the single-origin height system.

\subsubsection{Reliability}

The reliability of the geodetic network refers to the controllability of observations, in other words, the ability to detect outliers and to estimate the impact of undetected outliers on an adjusted solution (Leick, 2004). For evaluating the reliability, the redundancy number and the marginally detectable biases (MDB) were computed by variances for the observation and the adjusted residuals. The redundancy numbers ranging between 0 and 1 represent an insight into the geometric strength of a network; the lower, the less sufficient the outliers are isolated (Ghilani, 2010). On the other hand, the MDBs are the biases that can only be detected as outliers with a certain probability at a specific significance level (Caspary, 2000 and Harvey, 2006). Figure 11 provides the results of the redundancy numbers and the MDB, whereas Table 6 tabulates the statistics. Since CASE-I's DoF is higher than that of CASE-II as given in Table 4, the redundancy numbers of the former are slightly larger, and CASE-I's MDBs are overall more elevated than those of CASE-II.

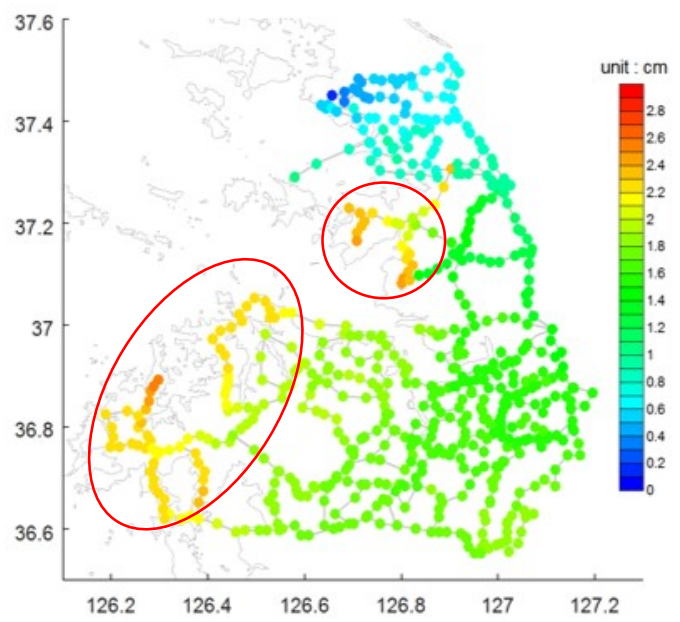

Figure 10: Absolute accuracy of the adjusted heights: the left is results of CASE-I while that of right is those of CASE-II

Table 5: Statistical summary of the absolute accuracy $(1 \sigma$, unit: $\mathrm{mm})$

\begin{tabular}{|c|c|c|c|c|}
\hline Case & Maximum & Minimum & Average & Standard Deviation \\
\hline I & 19.7 & 1.5 & 13.3 & \pm 3.8 \\
\hline II & 25.6 & 1.6 & 16.1 & \pm 4.7 \\
\hline
\end{tabular}



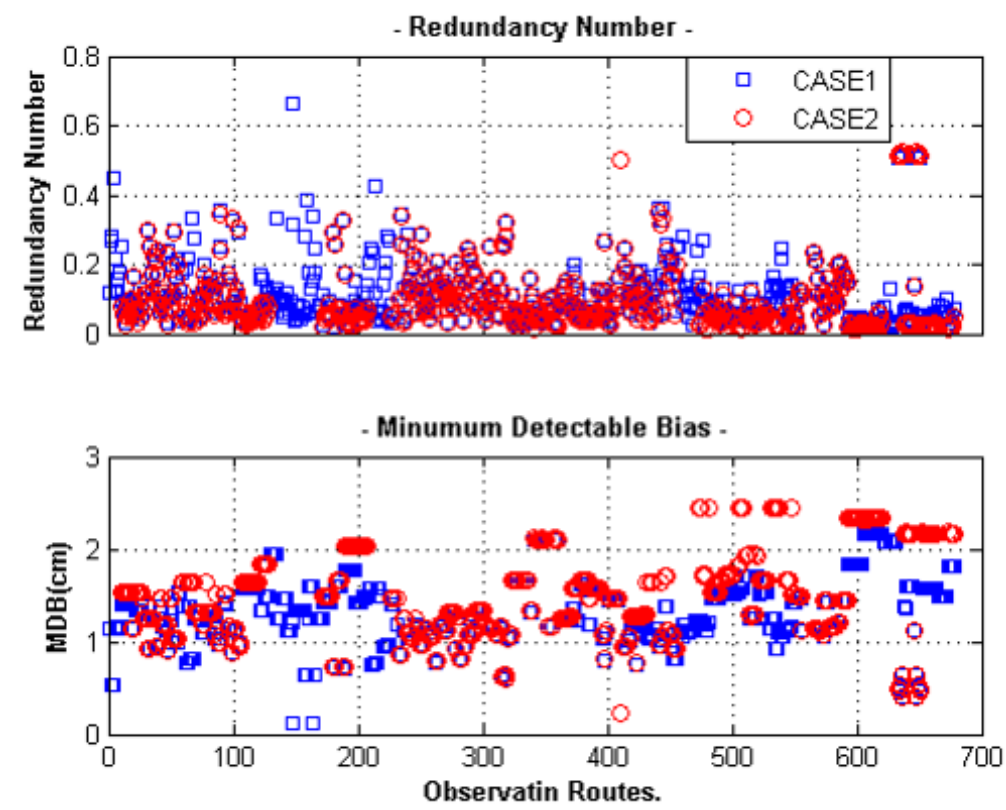

Figure 11: Reliability comparison of the final adjustments

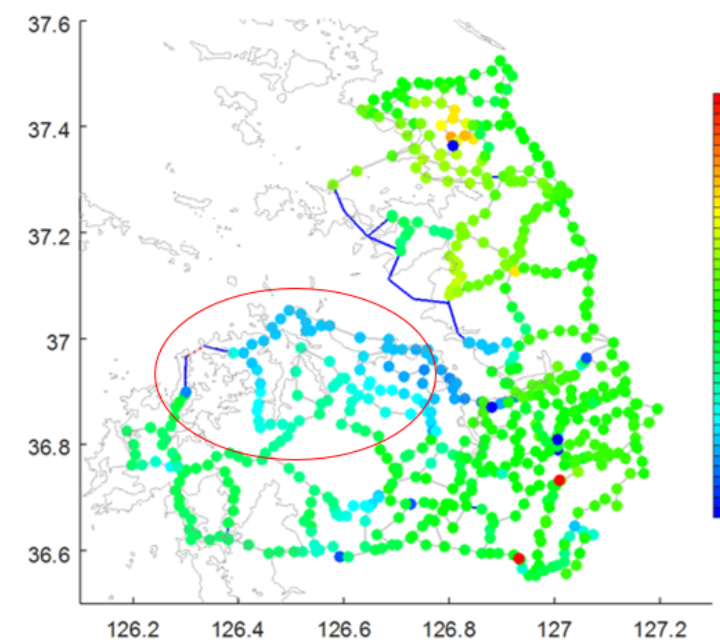

(a) CASE-I and Published heights

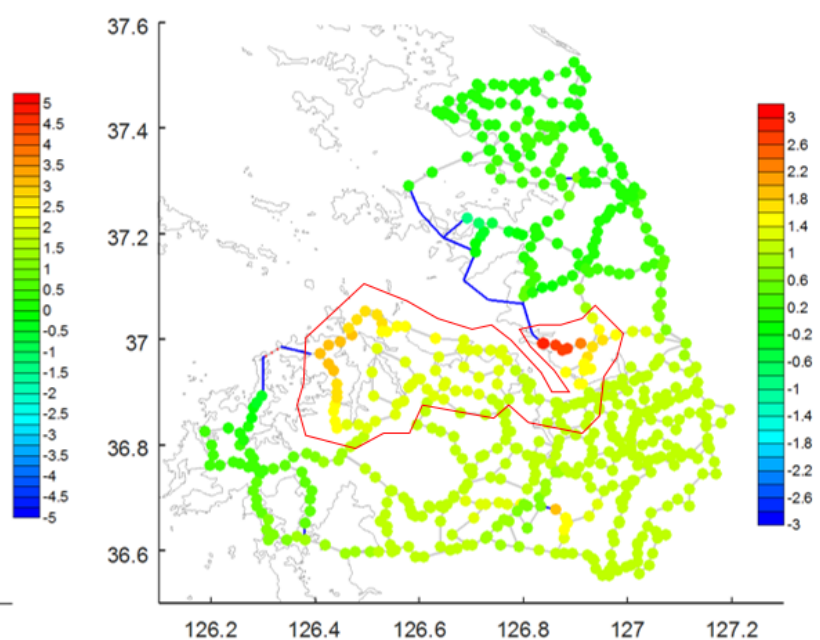

(b) CASE-I and II

Figure 12: A comparison of the adjusted heights

Although the outcomes indicate that the reliability of the CASE-I is increased by the newly installed UCPs as well as the surveying in this study, the geometric strength is still too low to sufficiently identify blenders as the redundancy numbers about 0.5 are generally required for a surveying network (Ghilani, 2010). This is, however, an indispensable drawback of the geodetic levelling due to the laborious field works. Furthermore, it is remarkable to note that the number of measurements of which the redundancy number is zero; CASE-1 is much smaller than that of CASE-II. Note that if the redundancy number is zero, the computed residual goes infinity, indicating the lack of measurement self-checking.

\subsubsection{Adjusted heights}

The adjusted heights of CASE-I have been compared to those published by NGII, and results are illustrated in the left map in Figure 12, which shows the influence of the UCPs-based levelling loops on the height estimation. It can be seen from results that the heights of the north area are somewhat high whereas those of the south are low, for instance, about $3.5 \mathrm{~cm}$ of bias around the circled area in the left map of the figure. This might be induced by the establishment of the additional loops along the west coastline. With consideration of the accuracy enhancement around the area depicted in Figure 10, the biases seem to be a positive influence on the adjusted heights.

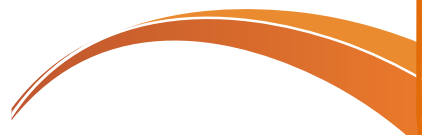


Toreover, it should be mentioned here that four points of the height differences are abnormally large as over $9 \mathrm{~cm}$. Note that these points are all the $2^{\text {nd }}$ order BMs which have been recently re-surveyed whereas the published heights are outcomes from observations in the mid-2000s. With this concern, as the monument displacements are suspected, further verification is highly recommended by field reconnaissance. Comparing the heights of CASE-I with the published, Table 7 reveals that the differences of the $1^{\text {st }}$-phase UCPs are more significant than those of the $2^{\text {nd }}$-order BMs. This is due to the fact that all the original loops of the former have been considerably amended by the new design strategy, and those of the latter are mostly kept.

The right map in Figure 12 depicts the height differences between the CASE-I and -II with some statistics in the lower part of Table 7. The more considerable differences are observed around the areas denoted by the solid polygon lines like the left map, but the magnitudes are limited. This means that the new network itself has more impacted on the height estimation, compared to the additional installation and survey. Furthermore, the height deviations of the $2^{\text {st }}$-phase UCPs and the $2^{\text {nd }}$-order $\mathrm{BMs}$ are more remarkable than the others as they are closely located to the new surveying area.

\section{Conclusions and Remarks}

This paper has introduced the multi-dimensional UCPs geodetic points, which is currently under construction in Republic of Korea, with a particular emphasis of the levelling network design and the geodetic adjustment. After addressing drawback of the current UCPs as a vertical control, a concept of the UCPs-based levelling network have been presented with a view to enhancing accuracy, reliability and accessibility. Depending on the technical circumstances, the three strategies have been proposed for designing the UCPs-based levelling network, such as the substitution of BMs by UCPs, the designation of BMs as UCPs, and the new installation of UCPs. By application of the concept, a pilot levelling network was composed of 621 points throughout the northwest region of Korea. After examining loop misclosures, a series of preliminary adjustments were made to troubleshoot blunders and determine a reference variance: one measurement was excluded; $\pm 1.7 \mathrm{~mm}$ and $\pm 3.4 \mathrm{~mm} / \sqrt{\mathrm{Km}}$ were defined as the reference standard deviations. Final adjustments have been performed twice, and the results indicated: (a) the new installation of the $2^{\text {nd }}$-phase UCPs and the additional observations achieved by this study overall improved the accuracy of the estimated heights but marginally enhanced the reliability; (b) the adjusted heights of the pilot network was biased to the published ones about $3 \mathrm{~cm}$ at maximum, which should be a positive impact on the height estimation in terms of accuracy due to the geometric enhancement.

NGII is the process of the project that nationwide installs the $2^{\text {nd }}$-phase UCPs and also spirit levelling campaigns together with gravimetric surveys, introducing the orthometric height system as a national standard. Furthermore, a nationwide GNSS and levelling network of the UCPs is processed to derive the multi-dimensional geodetic coordinates (i.e., latitude, longitude, and ellipsoidal and orthometric height); hence the UCPs network supersedes the legacy geodetic networks (i.e., triangulation and levelling network) and plays a backbone in the national geodetic infrastructure.

\section{Acknowledgement}

This research has been financially supported by the National Geographical Information Institute of Korea (NGII) in 2018. This paper is an extension of a presentation made at FIG Working Week, Hanoi, Vietnam, 22 ${ }^{\text {nd }}-26^{\text {th }}$ April 2019.

\section{References}

Bae, T. S., Kwon, J. H. and Hong, C. K., 2011, Topographic Optimal Network Design of the Unified Control Points in Korea. Earth Planets Space, Vol. 63, 411-418.

Bomford, B. G., 1962, Geodesy, 2nd edition, (Oxford: Clarendon Press)

Caspary, W. F., 2000, Concept of Network and Deformation Analysis, Monograph 11, School of Geomatic Engineering. The University of New South Wales, Australia, 183.

Cross, P. A., 1994, Advanced Least Squares Applied to Position Fixing, Working Paper No. 6. School of Surveying, University of East London, London, UK, 205.

Ghilani, C. D., 2010, Adjustment Computations: Spatial Data Analysis. 5th edition, (Hoboken, New Jersey: John Wiley \& Sons)

Harvey, B., 2006, Practical Least Squares and Statistics for Surveyors, Monograph 13. School of Surveying and Spatial Information Systems, The University of New South Wales, Australia, 332.

Lee, Y. J., Lee, H. K., Jeong, G. H. and Cha, S. H., 2008, Korean Geodetic Datum 2002 (KGD2002): Nationwide GPS Network Densification. FIG Working Week 2008, Stockholm, Sweden, 1419 June. 
eick, A., 2004, GPS Satellite Surveying. 3rd Edition, John \& Sons, Inc., (Hoboken, New Jersey: John Wiley)

NGII, 2006, A Study on the Korean Geodetic Network Adjustments, Project Report, (Republic of KOREA: National Geographical Information Institute of Korea).

NGII, 2013, A Study on Cost-Benefit Analysis and Maintenance of Geodetic Control. Project Report, (Republic of KOREA: National Geographical Information Institute of Korea).
NGII, 2014, A Study on the Establishment of a NextGeneration Geodetic Control Network. Project Report, (Republic of KOREA: National Geographical Information Institute of Korea).

NGII, 2015, Strategic Implementation Plan for a New National Spatial Reference Frame. Project Report, (Republic of KOREA: National Geographical Information Institute of Korea).

Steeves, R. R., 2015, GeoLabPX5 Guide. (Canada: BitWise Idea Inc.). 